\title{
Quantum Optimization of Resource Distribution Management for Multi-Task, Multi-Subtasks
}

\author{
Sara El Gaily and Sándor Imre
}

\begin{abstract}
This paper proposes a new optimization strategy for resource distribution management based on a quantum algorithm, as a way to reduce the computational complexity in finding the optimum deployment scenario, taking into consideration the required conditions and constraints of the resource distribution system. We show that the quantum method computes the results in minimum time and outperforms on the other classical algorithms in terms of computational complexity.
\end{abstract}

Index Terms -quantum computing; resource distribution management; quantum extreme value searching algorithm; quantum existence testing; computational complexity.

\section{INTRODUCTION}

\section{A. Motivation}

The first question that comes to the mind of the reader is how the quantum optimization methods may increase the performance system of resource distribution management process and how it will be used in resource management as a way to reduce the computational complexity in finding the optimum deployment scenario. What are the fundamental differences between a classical computer and quantum computer which can lead to choosing the quantum strategy as a future alternative solution for the resource distribution management model?

\section{B. Quantum Computing Overview}

In fact, quantum computer's functionality and conception work based on the laws of quantum mechanics. There is a large list of differences between the quantum computer and classical computer. First of all, classical computer functionality works based on the laws of classical mechanics, it performs calculations relying on the basic unit of information zeros and ones ( 0 and 1$)$, while quantum computer uses qubits which can take superposition of states at the same time [1], furthermore, quantum computer outperforms with high speed than the binary computer, as well as it can solve computational problems with

A preliminary version of this paper has been presented at the 2019 42nd International Conference on Telecommunications and Signal Processing (TSP) [15].

The research was partially supported by the National Research Development and Innovation Office of Hungary (Project No.2017-1.2.1-NKP-2017-00001), by the Hungarian Scientific Research Fund. The research reported in this paper was supported by the BME- Artificial Intelligence FIKP grant of EMMI (BME FIKP-MI/SC)".

Department of Networked Systems and Services, Budapest University of Technology and Economics. / Müegyetem. 3-9, Budapest, 1111, HUNGARY. Email: \{elgaily, imre\}@hit.bme.hu. low computational complexity, maximum accuracy, and short circumstance).

If we assume that a large number of binary computers can combine their efforts and overcome this gap, they cannot reach the performance level of a quantum computer. Quantum computing and information have important quantum algorithms that solve important computational problems which do not seem to be possibly solved by a classical method, for example, the most known actually are the quantum Fourier transform which is used to solve factoring and discrete logarithm problems, and its fascinating advantage to make the communication over a quantum channel more secure, and the quantum search algorithm [1][2] the so-called Grover's algorithm [3][4] which uses fewer steps than its classical counterpart to find a certain entry in an unsorted data with more accuracy, speed and less time.

\section{Establishing context and the importance of the research topic}

Resource distribution management must be designed to be highly reacting fast with maximum accuracy performance to any unpredictable task workload, as it is known, for tasks with fixed running time require more computation in a real-time system, since they are executed at a constant rate. In order to rationally use resource computing as a way to reduce the computational complexity in finding the best optimum deployment scenarios under the imposed constraints, we resorted to handling this problem by using an approach based on the quantum method. This study provides an important opportunity to improve the efficiency of using system resources by exploiting quantum computing methods and concepts.

Suppose all classical machines that are working on classical laws of classical physics will disappear from our world and be replaced by quantum computers, so there will be an intensive need for developing new adaptive models. From a resource distribution management point of view, if the real-time decisionmaker will be replaced by a quantum method, so, the way becomes open to think how to implement a resource distribution management model with the new device since new hardware technique requires a new resource distribution process modeling.

\section{A Brief Synopsis of the Relevant Literature}

It is difficult to relate the proposed strategy to other work in the literature because the proposed resource distribution management based on quantum optimization is a new contribution. So, we will try to give approximately and generally 
the recent works that have been proposed in this field. Periodic activities have the major computational demand in many realtime applications since they provide a simple way to enforce timing constraints through rate control [5], there has been a greater interest in proposing new techniques appropriate in using system resources of fixed real-time tasks. In [6], the Quality of service-based resource distribution which addresses the problem of distributing a bandwidth portion among services merged with the distribution algorithm in order to decrease computational complexity. In [7], a proposed solution for finding the optimal task periods for practical problems with a remarkable speedup by exploiting the concept of the exact feasibility region of the space. In [5]-[8], the elastic task model (ETM) was taken as an efficient mechanism for controlling the quality of service of the system as a function of the current load, the ETM is extremely useful for supporting both multimedia systems and control applications in which the execution rate of some computational activities have to be tuned as a function of the current system state. On the other hand, some recent works were using the Hungarian combinatorial algorithm as a tool for assigning tasks, for example, in [9] for multi-task to multi-worker allocation based on the demand distribution model, or, in [10], a decentralized task allocation algorithm based on the Hungarian approach. In [11], for channel allocation problem over a frequency-selective channel. Moreover, for a multicasting problem, two heuristics algorithms [12], Farthest First and Nearest First based were applied to minimize the number of used wavelengths. In [13], the orchestration algorithm was used in a heterogonous cloud environment to minimize the usage of computing resources.

This study is an extension of the previously published work, in [14] we have been started from a simple resource distribution management model, for one task generator: $(a)$ demonstrating analytically that the quantum solution is more efficient by comparing the computational complexity and distribution uniformity of the quantum solution with the randomized, exhaustive and sequence methods, $(b)$ showing the importance of the quantum solution, a simulation environment of the proposed optimization of distribution system was constructed and compared to two reference distribution systems which follow the randomized and sequence strategies. In [15], we have set up carefully the system parameters of the quantum algorithm with respect to the proposed resource distribution model (it contains one task generator). Furthermore, we discussed the most important parameters and derived the appropriate approximation formulas if different computation units are allowed in the system.

\section{E. Contribution}

This paper provides a new and comprehensive study on reducing the computational complexity of a distribution problem, using a system of multiple task generators which dissociate each task to several subtasks, integrating resource distribution model in the quantum system-level framework is not straight-forward and may need to a careful configuration of its parameters. The quantum approach will improve the speed of computation as well as the accuracy in selecting the best result, allowing the movement from an $O(d)$ computational complexity to $O\left(\log _{2}(T) \log _{2}{ }^{3}(\sqrt{d})\right)$.
The main questions addressed in this paper are:

- From a computational complexity point of view, how can we use the quantum searching method in resource distribution management? And how much is it efficient?

- From an engineering point of view, how can we set up the stochastic parameters of the quantum logarithm search according to the given resource distribution model?

\section{F. Organization}

The remaining part of this paper is organized as follows: Section II begins by describing a resource distribution management model with multi-task and multi-subtasks handling with one optimization metric. Then we will discuss how can we apply the quantum optimization algorithm to improve the efficiency of system resources from a computational complexity point of view, after that, we will demonstrate how the quantum approach is an efficient computational infrastructure tool by comparing it with the classical approach method, finally we will set up the system parameters of the quantum algorithm of the resource distribution management model. Section III concludes the paper.

\section{QuANTUM Resource Distribution MANAGEMENT \\ OPTIMIZATION IN MULTI-TASK AND MULTI-SUBTASKS}

In the resource distribution model, the uniformity distribution metric is a perfect standard measurement for checking whether resource utilization is balanced or not, in our study, we will rely fundamentally on the relative load variance of the system for measuring the uniformity distribution degree. For a large number of resources, it is difficult to compute classically the overall possible deployments which fit the optimum distribution, the solution is to exploit the power of quantum approach which will guarantee a high result in computational complexity reduction as well as accuracy performance.

In order to not confuse the reader, before explaining how the quantum method works in the resource distribution management model, first, we will give an overview of the quantum algorithm.

\section{A. Quantum Extreme Value Searching Algorithm Optimization}

The quantum extreme value searching algorithm QEVSA [16] combines the well-known logarithmic binary search algorithm which is originally intended for searching a given item in a sorted database [17] with the quantum existence testing, it is represented in the algorithm as QET [18]-[19]. Quantum existence testing is a special case of quantum counting, it focuses on checking the existence of a given entry in the database rather than in determining the number of existent entries. The QEVSA aims to find the extreme value (minimum or maximum point) of a point called cost function or database. Moreover, the power of quantum existence testing is derived from the quantum phase estimation which makes it outperforms better than the other algorithms, this technique produces an algorithm which keeps the efficiency of the binary search while processing an unsorted database. The proposed algorithm is introduced in [16]. 


\section{B. Resource Distribution Management Model with multi-task and multi-subtasks}

For a fixed reservation requirement, i.e., the contracted capacity amount used for the task, any available capacity left unreserved cannot be reused by other tasks. maximizing and improving the resource utilization requires using multi-subtask model, i.e., any task has subtasks. As aforementioned, the high utilization of computing resources and huge demand for computation leads to a search for efficient and less operational costs with respect to the required quality of service, for this purpose, we proposed a resource allocation model for running the workload. In order to model the general resource distribution management system, we divided the functionalities into three main blocks:

Multiple task generator: Let $u$ denote the number of task type generators, each task generator has its own task arrival time distribution and produce identical tasks, let the number of subtasks generated by the $l^{\text {th }}$ task generator be denoted by $g_{l}$ and the total number of subtasks type generated by all the task generators is denoted by $W$. Note that every subtask type $v$ has a fixed running time $p_{v}$ and the memory requirement for the subtask type $v$ is $\Delta_{v}$.

Decision maker: It is responsible for the deployment of the subtasks among the computing units, later we will explain the role of the quantum approach in reducing the computational complexity of the task deployment.

Computing units: Let $c$ be the number of computing units used to serve the subtasks, the computing units may have different theoretical capacities, such as the $i^{\text {th }}$ unit which has $s_{i}$ as a theoretical capacity, let the number of running subtasks from type $v$ on-the $i^{t h}$ unit is denoted by $N_{i v}^{b}$. Fig. 1 represents the resource distribution management structure.

In compliance with what has been already discussed, we have chosen to distribute uniformly the tasks among the computing units, the variance of the relative load in the system is used as a measurement metric for uniformity performance. In the case of optimal task distribution, if the variance of the relative load tends to zero, then the resources are distributed uniformly, otherwise, they are not, the formula of the relative variance is as follows,

$$
\sigma^{2}=\frac{1}{c} \sum_{i=1}^{c}\left(\bar{b}-\frac{\sum_{v=1}^{W} N_{i v} \Delta_{v}}{s_{i}}\right)^{2},
$$

Where $\bar{b}$ is the average of the relative load of the system. Later, we will see how the variance is employed in the quantum algorithm for searching the optimum deployment scenario.

\section{How to Use Quantum Extreme Value Searching in Resource Distribution Management}

The reader should bear in mind that for the proposed distribution model, this study did not discuss the resource requirement scheme implementation because the quantum existing testing is a special form of quantum phase estimation and quantum gate circuit structure is well known, in this paper, the modification will be only in the quantum extreme value searching algorithm.

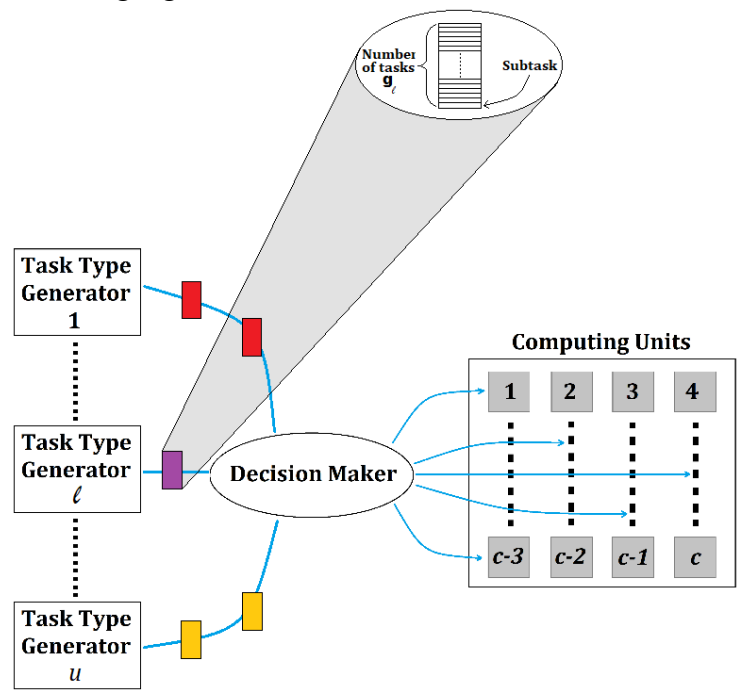

Fig.1: Resource distribution management architecture

Conserving the uniformity load of the system implies finding the optimum scenario that corresponds to the minimum variance, thus, in this case, we use the quantum extreme value searching algorithm as a minimum searching algorithm. What makes this proposed quantum approach special, is that it handles the database as a function, i.e., variance. The corresponding quantum algorithm according to the desired resource distribution model is given as follows,

1. We start with $S=0: \sigma^{2}{ }_{\min 1}=\sigma^{2}{ }_{\min 0}, \sigma^{2}{ }_{\max 1}=$ $\sigma_{\max 0}^{2}$, and $\Delta \sigma^{2}=\sigma^{2}{ }_{\max 0}-\sigma^{2}{ }_{\min 0}$

2. $S=S+1$

3. $\sigma_{\text {med } S}^{2}=\sigma_{\min S}^{2}+\left[\frac{\sigma^{2}{ }_{\max S}-\sigma^{2}{ }_{\min S}}{2}\right]$

4. $\quad$ lag $=Q E T\left(\sigma_{\text {med } S}^{2}\right)$ :

- If $f$ lag $=Y e s$, then $\sigma^{2}{ }_{\max S+1}=\sigma^{2}{ }_{\operatorname{med}}{ }^{2}, \sigma^{2}{ }_{\min S+1}=\sigma^{2}{ }_{\min S}$

- Else $\sigma^{2}{ }_{\max S+1}=\sigma^{2}{ }_{\max S}, \sigma^{2} \min S+1=\sigma^{2}$ med $S$

5. If $\mathrm{S}<\log _{2}(T)$, then go to 2, else stop and $y_{\text {opt }}=\sigma^{2}{ }_{\text {med } S}$

The maximum number of the necessary steps to run the logarithm search $T$ depends on two parameters which are the step size of the search which is according to the proposed distribution model is the minimum distance between variances of two scenarios $\alpha$ as presented in (2) and the size of the region of the variance's values $\Delta \sigma^{2}=\sigma^{2}{ }_{\max }-\sigma^{2}{ }_{\min }$, the expression of $T$ is illustrated in (3),

$$
\begin{aligned}
& \alpha=\min _{\forall V_{i}, V_{j}}\left|\left(\sigma_{V_{i}}^{2}-\sigma_{V_{j}}^{2}\right)\right|, \\
& T=\frac{\sigma_{\text {max }}^{2}-\sigma_{\text {min }}^{2}}{\alpha},
\end{aligned}
$$


Where $V_{i}$ and $V_{j}$ referred to two different assignment scenarios. Note that the stochastic variable $\alpha$ depends on many parameters such as the number of presented subtasks, processing time of each arrival task type distribution, the number of presented computing resources, etc.

Note that, integrating the resource distribution model in the framework of quantum system-level is not straight-forward and may need a careful configuration to its parameters. We are interested in providing a rigorous mathematical demonstration for bounding $\alpha$ based on the performance specifications of the proposed resource distribution model, as it is required in real physical implementation.

In the current subsection, we answered the fundamental question on how to apply the quantum method in the resource deployment system. In the next subsection, we will present an analytical comparison between the proposed quantum strategy and the classical counterpart.

\section{Analytical comparison between the computational} complexity of the quantum and the classical strategy.

The quantum minimum searching algorithm is used as a tool to reduce the computational complexity for selecting the optimum deployment scenario, the time complexity of the quantum method of the entire system is $O\left(\log _{2}(T) \log _{2}{ }^{3}(\sqrt{d})\right)$, it depends on the computational complexity of the quantum existence testing function $\log _{2}{ }^{3}(\sqrt{d})$ and the logarithm search of the quantum algorithm $\log _{2}(T)$, where $d$ refers to the number of possible deployment scenarios, this quantum technique uses fewer steps than the other searching methods like heuristic and randomized algorithms [20][21], etc.

To calculate the maximum number of steps $T$ which are necessary to run the logarithm search of the quantum algorithm for every bunch of new coming subtasks to the system, the real problem lies in calculating properly the value $\alpha$ at every task arrival (which means that the value of $\alpha$ changes for every new coming task), so in order to not confuse the reader we will denote this repeatedly computed value of $\alpha$ by $\alpha_{\text {actual }}$. Finding the value of $\alpha_{\text {actual }}$ requires determining the minimum distance for any two different load distributions, mathematically expressed as $\alpha_{\text {actual }}\left(V_{i}, V_{j}\right)=\min _{\forall \boldsymbol{V}_{\boldsymbol{i}}, \boldsymbol{V}_{\boldsymbol{j}}}\left|\left(\boldsymbol{\sigma}_{\boldsymbol{V}_{\boldsymbol{i}}}^{2}-\boldsymbol{\sigma}_{\boldsymbol{V}_{\boldsymbol{j}}}^{2}\right)\right|$. The computational complexity for finding $\alpha_{\text {actual }}$ is $O\left(\frac{d(d-1)}{2}\right)$, in the worst case, $d=c^{g_{l}}$, where $g$ is the number of subtasks in the arrived task from type $l$, it is noticeable that computing the value of $\alpha_{\text {actual }}$ is computationally hard.

Instead of calculating $\alpha_{\text {actual }}$ at each arrival task, the alternative solution is to compute in advance the global nonzero minimum of $\alpha$, denoted by $\alpha_{\text {global }}$, before starting the operation of the system, such that $\alpha_{\text {global }}=$ $\min _{\forall S_{c h}^{i} S^{j}} \alpha_{\text {local }}\left(S_{c h}^{i}, S_{c h}^{j}\right)$, where $\alpha_{\text {local }}$ means minimization over all possible load configurations of changed unit sets $S_{c h}^{i}$ and $S_{c h}^{j}$ belonging to the distributions $V_{i}$ and $V_{j}$. Because a certainly changed unit set fits many distributions, it is enough to define the previous formula with $i$ and $j$. The expression of the value of $\alpha_{\text {local }}$ is influenced only by a load of changed computing units, as we will see the proof later in the next subsection E, while unchanged computing units have no effects on $\alpha_{\text {local }}$.

Investigating all unit set pairs to calculate $\alpha_{\text {global }}$ we need to compute all $\alpha_{\text {local }}$ which are related to unit sets, assuming that the number of computing unit types is $\theta$, taking advantage of the previous statement of $\alpha_{\text {global }}$, we conclude that the number of possible distributions $d^{\prime}$ is less or equal than $\theta^{g_{\max }}$ where $g_{\max }$ is the number of arrival subtasks, which means that the computational complexity at this stage is $O\left(\frac{d^{\prime}\left(d^{\prime}-1\right)}{2}\right)$, this computation complexity is significantly less than the computation complexity of $\alpha_{\text {local }}$, the disadvantage of this minimization will create an increase in the maximum number of steps, but the quantum approach can handle the logarithm complexity of a large number of scale values because it will not increase significantly the complexity of the system.

The present subsection covers a comparison between the classical and the quantum approach. The question was how to reduce the computational complexity for the setup of the repeated changing value of $\alpha$ for any coming task, The alternative solution was to compute the global value $\alpha_{\text {global }}$ by exploiting the value of $\alpha_{\text {local }}$. The next subsection addresses the formulation of $\alpha_{\text {local }}$ taking into consideration the resource distribution parameters.

\section{E. Setting up the system parameter of the resource distribution management model with multi-task and multi- subtasks}

As already mentioned, in order to fully exploit the potential of the quantum minimum searching algorithm, it is necessary to configure properly the parameters of the quantum method according to the characteristics of the resource allocation model. As aforementioned, the parameter $T$ depends on $\Delta \sigma^{2}$, in the worst case, the value of $\sigma_{\max }{ }^{2}$ is 0.25 and the value of $\sigma_{\min }{ }^{2}$ will be always 0 . Thus $\Delta \sigma^{2}=\sigma_{\max }{ }^{2}$.

A more interesting approach addressed in this paper consist of providing concrete configuration of the quantum algorithm according to the mathematical formula given in (2), for the sake of the minimum distance between variances of two scenarios $\alpha_{\text {local }}$, so, it is necessary to find a manageable expression of $\left(\boldsymbol{\sigma}_{\boldsymbol{V}_{\boldsymbol{i}}}^{2}-\boldsymbol{\sigma}_{\boldsymbol{V}_{\boldsymbol{j}}}^{2}\right)$.

Let $x_{k}, b_{k}$ and $\bar{b}$ be respectively the total of the $\mathrm{k}^{\text {th }}$ unit load before receiving new subtask types, the relative load of the $\mathrm{k}^{\text {th }}$ unit, and relative load average as it is shown respectively in (4), (5) and (6),

$$
x_{k}=\sum_{v=1}^{W} N_{k v}^{b} \Delta_{v},
$$

where $N_{k v}^{b}$ refers to the number of subtask type $v$ in the $\mathrm{k}^{\text {th }}$ computing unit,

$$
b_{k}=\frac{x_{k}}{s_{k}}
$$




$$
\bar{b}=\frac{1}{c} \sum_{k=1}^{c} b_{k} .
$$

The problem was in formulating a general expression of the minimum distance between variances of two scenarios $\left(\boldsymbol{\sigma}_{\boldsymbol{V}_{\boldsymbol{i}}}^{2}-\right.$ $\boldsymbol{\sigma}_{V_{j}}^{2}$ ), i.e., for a given distribution scenario which fits to assigning only one subtask to a given unit. For this purpose, we considered that the load status of the computing units before and after the task deployment is given respectively by the $V^{b}$ (7) and $V^{a}$ matrices, their rows represent the computing units and the columns denote the subtasks type (8). In order to find the expression of the minimum variance between two different scenarios, the load of the new subtasks type should be taken into account, let's denote the set of computing units receiving the new subtasks by $S_{c h}$ and the remaining set of computing units by $S_{u n}$, such that $S_{c h} \cup S_{u n}=S_{T}$. For the sake of a simple notation that describes all the possible deployment scenarios of the new subtasks, we use $V_{i}$ matrix, its rows represent the computing units and the columns denote the subtask types as it is expressed in (8),

$$
\begin{gathered}
V^{b}=\left[\begin{array}{cccccc}
N_{11}^{b} & \cdots & \cdots & \cdots & \cdots & N_{1 W}^{b} \\
\vdots & \ddots & \ddots & \cdots & \cdots & \vdots \\
\vdots & \ddots & \ddots & \ddots & \cdots & \vdots \\
\vdots & \cdots & \ddots & \ddots & \ddots & \vdots \\
\vdots & \cdots & \cdots & \ddots & \ddots & \vdots \\
N_{c 1}^{b} & \cdots & \cdots & \cdots & \cdots & N_{c W}^{b}
\end{array}\right], \\
V_{i}=\left[\begin{array}{cccccc}
N_{11}^{V^{i}} & \cdots & \cdots & \cdots & \cdots & N_{1 W}^{V^{i}} \\
\vdots & \ddots & \ddots & \cdots & \cdots & \vdots \\
\vdots & \ddots & \ddots & \ddots & \cdots & \vdots \\
\vdots & \cdots & \ddots & \ddots & \ddots & \vdots \\
\vdots & \cdots & \cdots & \ddots & \ddots & \vdots \\
N_{c 1}^{V^{i}} & \cdots & \cdots & \cdots & \cdots & N_{c W}^{V^{i}}
\end{array}\right] .
\end{gathered}
$$

The relation between the load status of the computing units before and after the task deployment is $V^{b}+V_{i}=V^{a}$

The relative load of the set of computing units receiving the new subtasks $S_{c}$ is denoted by $\boldsymbol{b}_{k \in S_{c h}}^{V^{i}}$ and the relative load of the remaining computing units $\boldsymbol{b}_{k \in S_{u n}}$ is formulated as follows,

$$
\left\{\begin{array}{l}
b_{k \in S_{c h}}^{V^{i}}=b_{k \in S_{c h}}+V_{i} \Delta \\
b_{k \in S_{u n}}=b_{k}
\end{array}\right.
$$

Taking into consideration the new deployment subtasks, the average of the relative load of the resource model is expressed by the formula (10), note that $\boldsymbol{P}=\left[\begin{array}{lll}S_{1} & \ldots & S_{W}\end{array}\right]$ and $\Delta=$ $\left[\begin{array}{lll}\Delta_{1} & \ldots & \Delta_{W}\end{array}\right]^{t}$,

$$
\overline{b_{V_{l}}}=\frac{1}{c} \sum_{k=1}^{c} b_{k}+\frac{1}{c} \boldsymbol{P} \boldsymbol{V}_{\boldsymbol{i}} \Delta .
$$

The relative load of the variance of the new deployment scenario is given as follows,

$$
\sigma_{V_{i}}^{2}=\frac{1}{c} \sum_{k=1}^{c}\left(\overline{b_{V_{l}}}-b_{k}^{V_{i}}\right)^{2} .
$$

In the end, we end up with the corresponding formula, which is expressed as follows,

$$
\begin{aligned}
& \boldsymbol{\sigma}_{V_{i}}^{2}-\boldsymbol{\sigma}_{V_{j}}^{2} \\
& +2\left[\sum_{k \in S_{c h}^{V^{i}}}\left(\frac{\sum_{v}^{W} N_{k v}^{V^{i}}}{S_{k}}\right)^{2}-\sum_{k \in S_{c h}^{V^{j}}}\left(\frac{\sum_{v}^{W} N_{k v}^{V^{j}}}{S_{k}}\right)^{2}\right. \\
& +\sum_{k \in=1}^{W}\left(\sum_{k v}^{b}\right)\left(\frac{\sum_{v}^{W} N_{k v}^{V^{i}}}{S_{k}{ }^{2}}\right) \\
& \left.\left.\left.\sum_{c h}^{W} N_{k v}^{b}\right)\left(\frac{\sum_{v}^{W} N_{k v}^{V^{j}}}{S_{k}{ }^{2}}\right)\right)\right]
\end{aligned}
$$

The value of $\alpha$ denotes the smallest distance between two different scenarios among all the possible scenarios in a database. $\alpha$ is illustrated in Fig. 2

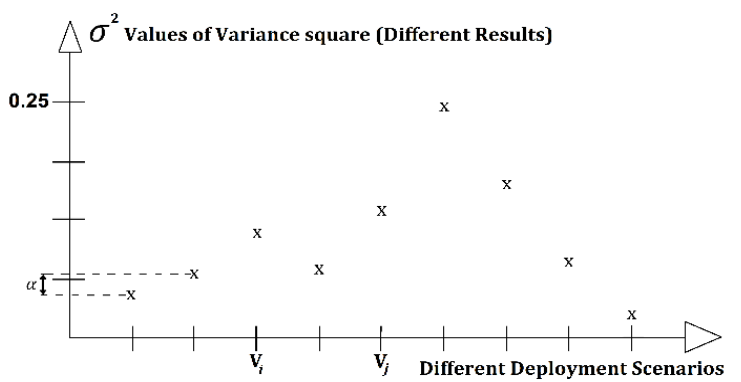

Fig.2: The horizontal axis presents all the possible deployment scenarios, while the vertical axis presents the borders of the variance square function (different results), each possible scenario corresponds to a variance value. Computing the value of $\alpha$ requires selecting the minimum distance between variances of two deployment scenarios $V_{i}$ and $V_{j}$.

It is important to mention that for any distribution, the difference between variances of two different scenarios depends only on the set of the computing units $S_{c h}$ that have been assigned a workload, not on all computing units.

Note that we considered that the number of incoming subtasks for both scenarios $V^{i}$ and $V^{j}$ are considered as fixed parameters for the system distribution, another important remark that we want to investigate is the non-zero $\alpha$. Furthermore, it is clearly noticeable that $\left(\sigma_{V_{i}}^{2}-\sigma_{V_{j}}^{2}\right)^{2} \geq 0$, in order to find the minimum places, it is enough to investigate $\left(\sigma_{V_{i}}^{2}-\sigma_{V_{j}}^{2}\right)^{2}=0$, this expression derives an important property of the minimum points (i.e., the variables $N_{k v}^{b}, v \in$ $\{1, \ldots, W\}$ are linearly dependent), in compliance with this result we conclude that the minimum places of the function $\left(\sigma_{V_{i}}^{2}-\sigma_{V_{j}}^{2}\right)^{2}$ are situated in a hyperplane.

In the general case, to determine the desired $\alpha$, it is needed to fulfill a certain number of restrictions determined by choosing the suitable range of the variables $N_{k v}^{b}, v \in\{1, \ldots, W\}$. The 
function $\left(\sigma_{V_{i}}^{2}-\sigma_{V_{j}}^{2}\right)$ is continuous, however, from resource distribution management point of view the variables $N_{k v}^{b}, v \in$ $\{1, \ldots, W\}$ must be integers. The first step is to assign an integer number different than zero to all the variables except one value, which corresponds to the subtask $\boldsymbol{v}=\omega$, this value could be $N_{k \omega}^{b} / k \in S_{c h}^{V^{i}}$ (or $k \in S_{c h}^{V^{j}}$ ), then computing the value of $N_{k \omega}^{b} /$ $k \in S_{c h}^{V^{i}}$ (or $k \in S_{c h}^{V^{j}}$ ), that corresponds to the remaining variables, here, at this stage, we have two options whether the value of $N_{k \omega}^{b} / k \in S_{c h}^{V^{i}}$ (or $k \in S_{c h}^{V^{j}}$ ) is an integer or not.

$1^{\text {st }}$ case: if $N_{k \omega}^{b} / R \in S_{c h}^{V^{i}}$ (or $R \in S_{c h}^{V^{j}}$ ) is an integer

The minimum distance between the variances of two scenarios $\alpha$ equals to zero and the values of the variables $N_{k \omega}^{b}$, $v \in\{1, \ldots, W\}$ are not appropriate solutions for $\alpha$, so, in this case, we can modify $N_{k \omega}^{b} / k \in S_{c h}^{V^{i}}$ (or $R \in S_{c h}^{V^{j}}$ ), by increasing or decreasing $N_{k \omega}^{b}$, and assigning the value of $N_{k \omega}^{b}+\mathbf{1}$ or $N_{k \omega}^{b}-\mathbf{1}$ to $N_{k \omega}^{b} / k \in S_{c h}^{V^{i}}$, (or assigning the value of $N_{k \omega}^{b}+\mathbf{1}$ or $N_{k \omega}^{b}-\mathbf{1}$ to $\left.N_{k \omega}^{b} / k \in S_{c h}^{V^{j}}\right)$.

The most important thing is to choose only one assignment which results in the minimum value of $\boldsymbol{\alpha}$, let us investigate the value of $f=\left(\boldsymbol{\sigma}_{\boldsymbol{V}_{\boldsymbol{i}}}^{2}-\boldsymbol{\sigma}_{\boldsymbol{V}_{j}}^{2}\right)^{2}$ when we assign the value of $N_{k \omega}^{b}+$ $\mathbf{1}$ or $N_{k \omega}^{b}-\mathbf{1}$ to $N_{k \omega}^{b}$, (or assigning the value of $N_{k \omega}^{b}+\mathbf{1}$ or $N_{k \omega}^{b}-\mathbf{1}$ to $\left.N_{k \omega}^{b} / k \in S_{c h}^{V^{j}}\right)$, using the previous result which stated that $\left(\sigma_{V_{i}}^{2}-\sigma_{V_{j}}^{2}\right)^{2} \geq 0$, we get to the following result,

$$
f_{N_{k \omega}^{b}+1}^{b}=f_{N_{k \omega}^{b}-1}
$$

The above expression states that the cross-section of the hyperplane is symmetric on the minimum places of $\left(\sigma_{V_{i}}^{2}-\right.$ $\left.\sigma_{V_{j}}^{2}\right)^{2}$ in its dimensional space.

$2^{\text {nd }}$ case: if $N_{k \omega}^{b} / k \in S_{c h}^{V^{i}}$ (or $R \in S_{c h}^{V^{j}}$ ) is not an integer

The function $\left(\boldsymbol{\sigma}_{\boldsymbol{V}_{\boldsymbol{i}}}^{2}-\boldsymbol{\sigma}_{\boldsymbol{V}_{j}}^{2}\right)$ has monotonous nature, consequently, the solution is to assign to $N_{k \omega}^{b} / k \in S_{c h}^{V^{i}}$ (or $k \in$ $S_{c h}^{V^{j}}$ ) the nearest integer, in this case, $\alpha$ does not equal to zero.

In this section, a mathematical framework was developed, which jointly determines the minimum distance between the variances of two scenarios $\alpha$.

\section{F. Simulation}

We have developed a simulator to show the efficiency of the quantum method compared to the randomized method. We will apply the optimized strategy for two metrics independently, the resource distribution system contains three elements; they are defined as follows:

One task generator: The tasks are exponentially generated, all the tasks have the same memory and energy requirement.

Decision-Maker: This component is responsible for selecting the best placement of the task, so, we will have two simulations, the first one is to assign tasks randomly to the computing units and the second one is to use the quantum algorithm.
Resources: The system contains 30 computing units, there are 3 types of these computing units, the following table presents the initial energy consumption of every computing unit type and their theoretical capacity, i.e., the energy consumed from computing units when it is working and not serving any task. The service time of each task is fixed, it equals $5 \mathrm{~s}$ and its memory requirement is also fixed, it equals $2 \mathrm{Kbit}$, the amount of the necessary power consumption to complete the serving of each task is 10 Watts.

\begin{tabular}{|c|c|c|c|}
\hline Units type & Type 1 & Type 2 & Type 3 \\
\hline Initial Energy & 150 & 300 & 450 \\
\hline $\begin{array}{c}\text { Theoretical } \\
\text { capacity }\end{array}$ & 10 & 20 & 30 \\
\hline $\begin{array}{c}\text { Number of } \\
\text { computing units }\end{array}$ & 30 & 40 & 30 \\
\hline
\end{tabular}

Table.1: the characteristics of computing units

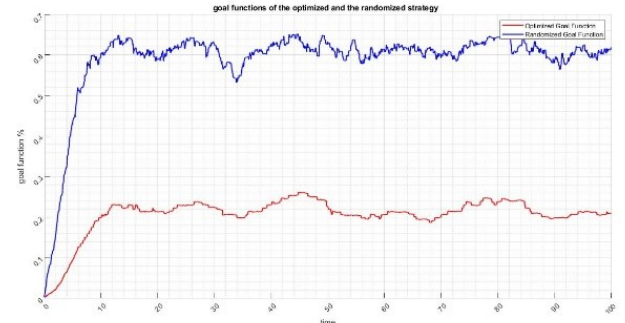

Fig.3: The overall energy consumption curves of the optimized (red line) and the randomized strategies (blue line), in case setting up the following parameters, the mean $=0.05$.

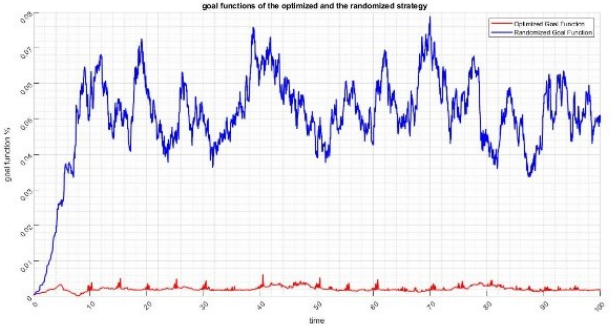

Fig.4: The variances curves of the optimized (red line) and the randomized strategies (blue line), in case setting up the following parameters, the mean = 0.05 .

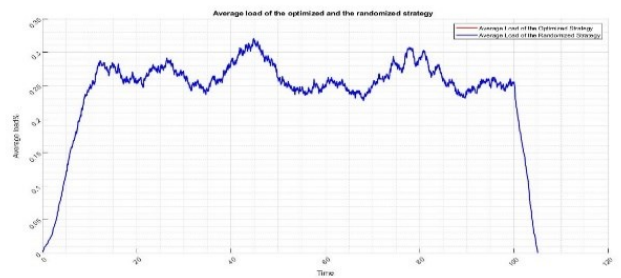

Fig.5: The average load curve of the optimized and the randomized strategies, in case setting up the following parameters, the mean $=0.05$.

According to Fig. 3 and Fig.4, the curves representing the total power consumption of the randomized algorithm are larger than the optimized method. As a conclusion, the optimized strategy has better results in finding the minimum overall power 
consumption and the best uniformity distribution than the randomized method during the whole simulation process.

\section{CONCLUSION}

This work addresses the problem of distributing task portions, i.e., subtasks, among different resource computing as a means to alleviate the computational complexity of selecting the optimum distribution scenarios, the main objective was to conserve the uniformity distribution load. The quantum minimum searching was our best choice for achieving optimal deployment results, which is dramatically influences in reducing the computational complexity of the system.

Also, we handled a general case that handles multioptimization metrics, as well as testing the proposed quantum approach by the simulation environment.

In the future work, the quantum algorithm will treat a model which handle more general case, multi-optimization metrics, as well as testing the proposed quantum approach by simulation environment, at the same time increasing the complexity problem by defining its nonlinear combination function, furthermore, we will try to implement constraints to our quantum algorithm.

\section{REFERENCES}

[1] S. Imre, F. Balázs. Quantum Computing and Communications - An Engineering Approach. John Wiley, England, 2005, 283 pp. DOI: $10.1002 / 9780470869048$.

[2] S. Imre, L. Gyongyosi. Advanced Quantum Communications: An Engineering Approach", 2012, DOI: 10.1002/9781118337462.

[3] Lov.K. Grover: "Quantum Computers Can Search Arbitrarily Large Databases by a Single Query" Vol 79, No 23, 1997. DoI: https://doi.org/10.1103/PhysRevLett.79.4709

[4] Lov.K. Grover: "A fast quantum mechanical algorithm for database search", InProc. STOC '96 Proc. of the Twenty-eighth annual ACM symposium on Theory of Computing, Philadelphia, Pennsylvania, USA, 1996, pp. 212-2019, DoI: 10.1145/237814.237866.

[5] Buttazzo G.C., Lipari G., Caccamo M., L. Abeni:” Elastic scheduling for flexible workload management". IEEE Transactions on Computers, Vol: 51, 2002. DoI: 10.1109/12.990127.

[6] Marau R., Lakshmanan K., Pedreiras P., Almeida L., Rajkumar R.:' Efficient Elastic Resource Management for Dynamic Embedded Systems", 2011 IEEE 10th International Conference on Trust, Security, and Privacy in Computing and Communications, 2011. DoI: $10.1109 /$ TrustCom.2011.135.

[7] Bini E., Natale M. Di: "Optimal task rate selection in fixed priority systems", 26th IEEE International Real-Time Systems Symposium (RTSS'05), 5-8 December. 2005, DoI: 10.1109/RTSS.2005.32.

[8] Buttazzo G.C., Lipari G., Abeni L.: "Elastic task model for adaptive rate control", InProc. 19th IEEE Real-Time Systems Symposium (Cat. No.98CB36279), 1998. DoI: 10.1109/REAL.1998.739754.

[9] Yu D.; Zhou Z., Wang Y.: "Crowdsourcing Software Task Assignment Method for Collaborative Development", IEEE Access, Vol 7, pp. 35743 - 35754. DoI: 10.1109/ACCESS.2019.2905054.

[10] Ismail S.; Sun L.: "Decentralized Hungarian-based approach for fast and scalable task allocation". International Conference on Unmanned Aircraft Systems (ICUAS), Miami, FL, USA, 2017, DoI: 10.1109/ICUAS.2017.7991447

[11] Bistritz I., Leshem A.: "Efficient and asymptotically optimal resource block allocation", IEEE Wireless Communications and Networking Conference (WCNC), 2018, DoI: 10.1109/WCNC.2018.8376960.

[12] Le, D. D, Molnár, M. and Palaysi, J., " Multicast Routing in WDM Networks without Splitters,", Infocommunication Journal, vol. 5, no. 2, pp. 1-10, June. 2013. DoI: 10.1109/MCOM.2014.6852098
[13] Szabo, M., Hajay, D. and Szalayz, M., " Cost-Efficient Resource Allocation Method for Heterogeneous," Infocommunication Journal, vol 10, no 1, March 2018.

[14] S.ElGaily, S. Imre. EVALUATION OF RESOURCE OPTIMIZATION BASED ON QUANTUM SEARCH. Hungarian Journal of industry and chemistry. 2019. DoI: 10.33927/hjic-2019-03.

[15] El Gaily S., Imre S., "Derivation of Parameters of Quantum Optimization in Resource Distribution Management". 42nd International Conference on Telecommunications and Signal Processing. DoI: 10.1109/TSP.2019.8769092.

[16] S. Imre: „Extreme Value Searching in Unsorted Databases Based on Quantum Computing". International Journal of Quantum Information. World Scientific 2005. Vol. 3. No. 1, pp. 171-176. DOI: $10.1142 / \mathrm{S} 0219749905000700$.

[17] Imre S: Quantum Communications - Explained for Communication Engineers IEEE COMMUNICATIONS MAGAZINE 51: 8 pp. 28-35., 8 p. (2013). DoI: 10.1109/MCOM.2013.6576335

[18] Donald K. (1998). Sorting and searching. The Art of Computer Programming. 3 (2nd ed.). Reading, MA: Addison-Wesley Professional. DoI: 10.1090/s0002-9904-1973-13173-8.

[19] Imre S., 'Quantum Existence Testing and Its Application for Finding Extreme Values in Unsorted Databases'. IEEE Transactions on Computer, Vol:56, no. 5, 2007. DoI: 10.1109/TC.2007.1032

[20] Ariffin N., Zin M., Norul S., Sheikh H., Faridatul N., Zainal A.: A Comparison of Exhaustive, Heuristic and Genetic Algorithm for Travelling Salesman Problem in PROLOG, International Journal on Advanced Science Engineering Information Technology. Vol 2, no.6, 2012. DoI: $10.18517 /$ ijaseit.2.6.244

[21] Hooker J.N.: Unifying Local and Exhaustive Search, Carnegie Mellon University, 2005. DoI: 10.1111/itor.12020

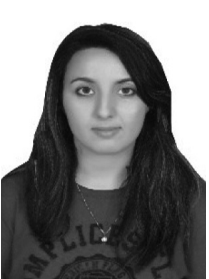

Sara El Gaily, a Ph.D. student at the Department of Networked Systems and services at the Budapest University of Technology and Economics (BME). She obtained her Master's degree in electrical systems and renewable energies at Hassan II University in 2017 in Casablanca, Morocco. She obtained her bachelor's degree in computer and industrial electronics in 2014. Her research interests are quantum computing and resource distribution management.

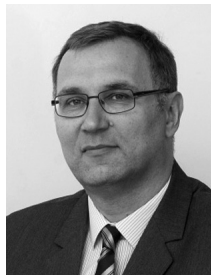

Sándor Imre [M'93] professor and Head of the Department of Networked Systems and services at the Budapest University of Technology and Economics (BME). He obtained dr. univ. degree in probability theory and statistics in 1996, a Ph.D. degree in 1999 and DSc degree from the Hungarian Academy of Sciences in 2007. He was elected the corresponding member of HAS in 2019. He acts as supervisor in the High-Speed Networks Laboratory since 1999. He is a member of the Doctoral Council of HAS. He was invited to join the eMobile Innovation Center of BME as an R\&D director in 2005. His research interests include mobile and wireless systems, quantum computing and communications. Especially he has contributions to different wireless access technologies, mobility protocols and their game-theoretical approaches, reconfigurable systems, quantum computing-based algorithms, and protocols. 